OPEN ACCESS

Edited by:

Bin Tang,

Hangzhou Normal University, China

Reviewed by:

Jae Park,

The University of Tennessee, Knoxville, United States

Pin-Jun Wan,

China National Rice Research Institute

(CAAS), China

*Correspondence:

Qiang Zhou

Isszhou@mail.sysu.edu.cn

${ }^{\dagger}$ These authors share first authorship

Specialty section:

This article was submitted to

Invertebrate Physiology,

a section of the journal

Frontiers in Physiology

Received: 18 June 2018 Accepted: 16 November 2018 Published: 29 November 2018

Citation:

Lu K, Zhang X, Chen X, Li Y, LiW, Cheng Y, Zhou J, You K and Zhou Q

(2018) Adipokinetic Hormone Receptor Mediates Lipid Mobilization

to Regulate Starvation Resistance in the Brown Planthopper, Nilaparvata lugens. Front. Physiol. 9:1730. doi: 10.3389/fphys.2018.01730

\section{Adipokinetic Hormone Receptor Mediates Lipid Mobilization to Regulate Starvation Resistance in the Brown Planthopper, Nilaparvata lugens}

Kai Lu ${ }^{1,2 \dagger}$, Xinyu Zhang ${ }^{1 \dagger}$, Xia Chen ${ }^{2}$, Yue $\mathrm{Li}^{2}$, Wenru $\mathrm{Li}^{2}$, Yibei Cheng ${ }^{2}$, Jinming Zhou ${ }^{1}$, Keke You ${ }^{1}$ and Qiang Zhou ${ }^{1 *}$

${ }^{1}$ State Key Laboratory of Biocontrol, School of Life Sciences, Sun Yat-sen University, Guangzhou, China, ${ }^{2}$ College of Life Sciences, Fujian Agriculture and Forestry University, Fuzhou, China

Lipid storage must be efficiently mobilized to sustain the energy demands during processes of exercise or starvation. In insects, adipokinetic hormone (AKH) and brummer lipase are well-known regulators of lipid mobilization. We recently demonstrated that brummer-dependent lipolysis regulates starvation resistance in the brown planthopper, Nilaparvata lugens, one of the most destructive rice pests. The present work investigated the roles of the AKH signaling system in lipid mobilization during the starvation process in N. lugens. NIAKHR is a typical G protein-coupled receptor (GPCR) and possesses high structure and sequence similarity to other insect AKHRs. Spatial and developmental expression profiles suggested that NIAKH is released from the corpora cardiaca to activate NIAKHR mainly expressed in the fat body. Starvation significantly induced the expression of NIAKH and NIAKHR, indicating a potential role of the $\mathrm{AKH}$ signaling system in starvation resistance. To reveal the functions of the $\mathrm{AKH}$ signaling system, a double-stranded RNA (dsRNA)-mediated knockdown of NIAKHR and NIAKH peptide injection was performed. The results show NIAKHR silencing decreased the levels of 1,2-diacylglycerol (DAG) in the hemolymph and increased triacylglycerol (TAG) levels in the fat body, whereas NIAKH injection led to a critical accumulation of DAG in the hemolymph and a severe reduction of TAG content in the fat body. Knockdown of NIAKHR resulted in prolonged lifespan and high levels of whole-body TAG, indicating an inability to mobilize TAG reserves during starvation. Conversely, the NIAKH injection reduced the survival and accelerated TAG mobilization during starvation, which further confirms the role of NIAKH in lipolysis. Moreover, NIAKHR silencing caused obesity in N. lugens, whereas NIAKH injection depleted organismal TAG reserves in vivo and produced a slim phenotype. These results indicate that lipid mobilization is regulated by the $\mathrm{AKH}$ signaling system, which is essential for adjusting body lipid homeostasis and ensuring energy supplement during starvation in N. lugens.

Keywords: adipokinetic hormone (AKH), adipokinetic hormone receptor (AKHR), lipid mobilization, starvation resistance, Nilaparvata lugens 


\section{INTRODUCTION}

The balance between lipid storage and mobilization is a critical characteristic of organismal energy homeostasis (Grönke et al., 2007). Most insects accumulate triacylglycerol (TAG), a strongly hydrophobic neutral lipid droplet with high energy content, as the primary lipid reserve for energy storage (Brown, 2001; Martin and Parton, 2006). TAG is mainly deposited in the fat body, an insect equivalent of adipose tissue, during periods of excessive food resources (Azeez et al., 2014). The ability to mobilize stored TAG reserves is important for the survival of insects under energy-demanding conditions. A tightly regulated balance between lipogenesis and lipolysis adjusts the TAG content in insects and matches acute energy needs in response to the fluctuation of environments (Grönke et al., 2007).

Two lipolytic systems, adipokinetic hormone (AKH)mediated lipolysis and brummer lipase-dependent lipolysis, were reported to be involved in the regulation of TAG mobilization in insects (Stone et al., 1976; Grönke et al., 2005). This contrasts with vertebrates, where only one lipolytic system that involves adipocyte triglyceride lipase (ATGL), a homolog of insect brummer, is known to metabolize TAG (Zimmermann and Zechner, 2004). In insects, the levels of hemolymph lipid and carbohydrates are regulated by $\mathrm{AKH}$, a peptide hormone which is thought to be functionally analogous to the mammalian glucagon (Lee and Park, 2004; Bharucha et al., 2008). AKH was first identified as an insect neurohormone that stimulates lipolysis and locomotor activity in Locusta migratoria (Mayer and Candy, 1969; Stone et al., 1976). To date, more than 60 different kinds of AKHs have been identified or predicted from genome sequencing projects as highly conserved peptide hormones with similar structural characteristics in insect species (Gäde and Marco, 2013). Functionally, insect AKHs have been shown to possess pleiotropic actions. For example, AKHs stimulate lipolysis of TAG into diacylglycerols (DAG) and the fat body-based conversion of glycogen into trehalose in response to starvation (Grönke et al., 2007). In addition to the energy-mobilizing activity, some other functions of AKHs have also been revealed, such as inducting foraging activity in starved Drosophila melanogaster (Lee and Park, 2004), stimulating midgut proteolytic activity in the flesh fly Sarcophaga crassipalpis (Bil et al., 2014) and playing an important role in oxidative stress (Bednářová et al., 2013). Recently, AKH signaling has also been shown to be strongly associated with insect reproduction (Lorenz, 2003; Lindemans et al., 2009; Attardo et al., 2012). AKH is synthesized and released from the corpora cardiaca (CC) into the hemolymph, then binds to a $\mathrm{G}$ protein-coupled receptor (GPCR) at the membrane of fat body cells, and eventually mobilizes lipid and carbohydrate reserves (Gäde and Auerswald, 2003; Caers et al., 2012; Gáliková et al., 2015).

The AKH receptor (AKHR) was first identified in the fruit fly D. melanogaster (Park et al., 2002) and the silkworm Bombyx mori (Staubli et al., 2002) as a rhodopsin-like GPCR with seven transmembrane-spanning alpha-helices, which is structurally and functionally analogous to the vertebrate gonadotropinreleasing hormone (GnRH) receptor (Lindemans et al., 2009). To date, AKHRs have been identified or predicted from genome sequencing projects in several other insect species (Supplementary Table 1). AKH signaling is achieved by binding peptide hormone with AKHR and then activating various cellular signaling pathways (Staubli et al., 2002). Insect AKH/AKHR signaling involves $\mathrm{Ca}^{2+}$ and cyclic adenosine monophosphate (cAMP) as intracellular messengers, and the signaling cascades of AKH/AKHR-stimulated lipolysis also involve the activation of protein kinase A (PKA) (Arrese et al., 1999; Van der Horst et al., 2001; Gäde and Auerswald, 2003). AKHR is ubiquitously expressed, however, it is highly accumulated in fat body, where AKH possibly functions in vivo. In the two-spotted cricket Gryllus bimaculatus, knockdown of AKHR led to the reduction of DAG and trehalose in the hemolymph whilst to elevated levels of TAG in the fat body (Konuma et al., 2012). Meanwhile, AKHR deficiency caused increased starvation resistance and decreased locomotory activity in the crickets (Konuma et al., 2012). Recently, it was shown that flies carrying AKHR mutations suffer from increased TAG and glycogen accumulation, supporting the idea that AKHR contributes to the lipolysis (Isabel et al., 2005; Bharucha et al., 2008). Along with these findings, AKHR mutant flies possess a phenotype of increased starvation resistance, which corresponds to the observation found in the AKH-deficient flies (Lee and Park, 2004; Grönke et al., 2007; Bharucha et al., 2008). Similar results were also obtained in Rhodnius prolixus (AlvesBezerra et al., 2016) and Bactrocera dorsalis (Hou et al., 2017), wherein the silencing of $A K H R$ reduced the levels of DAG and trehalose in the hemolymph and caused TAG accumulation in the fat body, which further underscores the central role of AKHR in insect lipolysis. The transcript expression of $A K H R$ was also detected in the reproductive tissues, supporting the idea that energy metabolism mediated by the AKH signaling system might be regulated to meet the demands for female reproduction. In the tsetse fly Glossina morsitans, knockdown of AKHR resulted in an accumulation of stored lipids during pregnancy and caused a severe reduction of fecundity (Attardo et al., 2012). Recently, AKHR was reported to modulate female sexual traits, fecundity and flight duration in B. dorsalis (Hou et al., 2017). Therefore, lipolysis mediated by the AKH/AKHR signaling system is not only required for basic physiological functions, but also for survival during prolonged starvation and reproduction in insects.

The migratory brown planthopper Nilaparvata lugens is one of the most destructive rice pests in Asia, with a strong reproductive capacity. Moreover, many field populations have developed high levels of insecticide resistance. Since the resistance and fecundity both heavily rely on proper lipid metabolism, lipolytic systems related to lipid mobilization might significantly contribute to planthopper outbreaks (Lu et al., 2018; Zhou et al., 2018a). In previous studies, we identified brummer, a lipase which regulates lipid mobilization and starvation resistance in the planthopper (Zhou et al., 2018b). However, insect lipolysis is under control of multiple regulatory systems, and $\mathrm{AKH}$-dependent mechanisms underlying the regulation of lipolysis have yet to be elucidated in this insect.

To reveal the role of $\mathrm{AKH}$ signaling pathway in lipid storage control, and to address the question of how these components orchestrate acute lipolysis in response to starvation, we identified the AKHR and analyzed its functions in vivo. 
We first investigated the evolutionary relationship of AKHR orthologs from other insect species, and then analyzed the expression patterns of $A K H$ (Tanaka et al., 2014) and AKHR in different tissues and developmental stages. Then, the expressions of AKH/AKHR signaling system components under starvation conditions were characterized. Finally, the roles of this system in lipid mobilization and starvation resistance were tested by $A K H R$ knockdown via RNA interference and $\mathrm{AKH}$ peptide injection. Our results suggest that the AKH/AKHR signaling system is indispensable for acute lipid mobilization and contributes to starvation resistance in the planthopper.

\section{MATERIALS AND METHODS}

\section{Insects and Sample Preparation}

Individuals of $N$. lugens were maintained at Sun Yat-sen University, which were originally sourced from a colony from the South China Agriculture University in September 2008 (Lu et al., 2015). Insects were reared on fresh rice seedlings (Taichung Native 1) and kept at $26 \pm 1^{\circ} \mathrm{C}$ with $65 \pm 5 \%$ humidity under a 16/8 h (light/dark) photoperiod condition. Newly emerged females were collected and kept isolated until used for treatments.

Tissues, including head, midgut, ovary, fat body and epidermis, were dissected from 30 females that were 3 days old to investigate tissue-specific expression profiles. The females were anesthetized on ice and dissected in a precooled phosphate-buffered solution (PBS, $140 \mathrm{mM} \mathrm{NaCl}, 2.7 \mathrm{mM} \mathrm{KCl}$, $10 \mathrm{mM} \mathrm{Na}_{2} \mathrm{HPO}_{4}$, and $1.8 \mathrm{mM} \mathrm{KH} \mathrm{PO}_{4} ; \mathrm{pH}$ 7.4) under a stereomicroscope (SMZ745, Nikon, Tokyo, Japan). Dissected tissues were placed in a $1.5 \mathrm{~mL}$ RNase-free centrifuge tube and immediately frozen in liquid nitrogen and stored at $-80^{\circ} \mathrm{C}$ until use.

Hemolymph was collected using a centrifugation method reported previously with moderate modification (Xu et al., 2006). Briefly, the females were anesthetized with carbon dioxide and the body wall of the thorax was pulled open using a tungsten needle. Then the punctured females were placed in a $0.5 \mathrm{~mL}$ RNase-free centrifuge tube with several small holes at the tube bottom. Next, the $0.5 \mathrm{~mL}$ tube was placed into a $1.5 \mathrm{~mL}$ RNasefree centrifuge tube and centrifuged at $9000 \times g$ at $4^{\circ} \mathrm{C}$ for 5 min. Thirty females were combined as one biological sample for hemolymph collection with three independent replicates.

\section{RNA Isolation, cDNA Synthesis and Cloning of NIAKHR}

Total RNAs from whole bodies or different tissues were isolated using TRIzol reagent (Invitrogen, CA, United States) according to the manufacturer's instructions. RNA integrity was confirmed by $1.5 \%$ agarose gel electrophoresis and RNA concentration was determined using a NanoDrop 2000C spectrophotometer (Thermo Fisher Scientific, West Palm Beach, FL, United States). In order to avoid genomic DNA contamination, total RNA was treated with RNase-free DNase I (Promega, Madison, WI, United States). A GoScript Reverse System (Promega) was used to synthesize the first-strand cDNA with $5 \mu \mathrm{g}$ of total RNA in a $20 \mu \mathrm{L}$ reaction mixture volume.
A partial cDNA sequence of putative $N I A K H R$ was identified from a transcriptome of $N$. lugens (SRX023419) by performing a tBLASTn search using $D$. melanogaster AKHR sequence (NP_995639) as a query, and then verified by searching against a N. lugens genome database (PRJNA177647) (Xue et al., 2014). The full-length of NlAKHR was amplified using a SMART ${ }^{\mathrm{TM}}$ RACE cDNA amplification kit (Clontech, Mountain View, CA, United States). Gene-specific primers were designed based on the cDNA partial sequence obtained as described above, and DNA polymerase GoTaq Master Mix (Promega) was used for RACE-PCR. Gene-specific outer primers and Universal Primer Mix (UPM) were used for the first round PCR (AKHR-F1 and UPM for $3^{\prime}$ RACE; AKHR-R1 and UPM for $5^{\prime}$ RACE) with the following amplification conditions: initial denaturation at $95^{\circ} \mathrm{C}$ for $2 \mathrm{~min}$, followed by 35 cycles at $95^{\circ} \mathrm{C}$ for $30 \mathrm{~s}, 50^{\circ} \mathrm{C}$ for $30 \mathrm{~s}, 72^{\circ} \mathrm{C}$ for $2 \mathrm{~min}$, and final extension at $72^{\circ} \mathrm{C}$ for $5 \mathrm{~min}$. Nested PCR was carried out with gene-specific inner primers and Nested Universal Primer (NUP) (AKHR-F2 and NUP for $3^{\prime}$ RACE; AKHR-R2 and NUP for $5^{\prime}$ RACE) with 35 cycles of amplification $\left(95^{\circ} \mathrm{C}\right.$ for $30 \mathrm{~s}, 50^{\circ} \mathrm{C}$ for $30 \mathrm{~s}, 72^{\circ} \mathrm{C}$ for $\left.1.5 \mathrm{~min}\right)$, and the diluted primary PCR amplification product was used as template. PCR products were separated by $1.5 \%$ agarose gel electrophoresis and purified (Tiangen, Beijing, China), subcloned into a pGEM-T easy vector (Promega) and transformed into E. coli $\mathrm{DH} 5 \alpha$ competent cells (Tiangen). The inserted cDNA was sequenced by Life Technologies Company (Guangzhou, China).

\section{Sequence Characterization and Phylogenetic Analysis}

The amino acid sequence of NlAKHR was deduced from the corresponding cDNA sequence using the ExPASy Proteomics translation tool, and the transmembrane domains were predicted using the TMHMM server 2.0 (Krogh et al., 2001). The potential signal peptide position was predicted using the SignalP 4.1 Server (Mccarthy et al., 2004). Protein sequences for AKHR were downloaded from GenBank and aligned by ClustalW algorithm and a phylogenetic tree was constructed by the MEGA 6 software using the Maximum Likelihood (ML) method with a bootstrap of 1000 replicates (Tamura et al., 2013).

\section{Reverse Transcription PCR (RT-PCR) and Real-Time Quantitative PCR (qPCR)}

RNA extraction and cDNA reverse transcription were performed as described above. Primers used for RT-PCR and qRT-PCR were designed by Primer 3 program (Untergasser et al., 2012) and are presented in Table 1. Partial cDNA fragments of NlAKHR, NlAKH, NlTUB (alpha 2-tubulin, FJ810204) and NlRPS11 (ribosomal protein S11, FJ810197) were amplified using GoTaq Master Mix (Promega) under the following conditions: initial denaturation at $95^{\circ} \mathrm{C}$ for $2 \mathrm{~min}$, followed by 30 cycles at $95^{\circ} \mathrm{C}$ for $30 \mathrm{~s}, 60^{\circ} \mathrm{C}$ for $30 \mathrm{~s}, 72^{\circ} \mathrm{C}$ for $45 \mathrm{~s}$, and final extension at $72^{\circ} \mathrm{C}$ for $5 \mathrm{~min}$. qRT-PCR was performed with a StepOnePlus ${ }^{\mathrm{TM}}$ Real-Time PCR system (Applied Biosystems) using the UltraSYBR Mixture (CWBIO, Beijing, China) under the following reaction conditions: one cycle for $10 \mathrm{~min}$ at $95^{\circ} \mathrm{C}$, followed by 40 cycles of $10 \mathrm{~s}$ at $95^{\circ} \mathrm{C}, 15 \mathrm{~s}$ at $60^{\circ} \mathrm{C}$ and $20 \mathrm{~s}$ at 
$72^{\circ} \mathrm{C}$. A melting curve analysis was performed at the end of each qPCR to check the amplification specificity and to rule out the possibility of primer-dimer formation. All PCR reactions were carried out in triplicate, and at least two technical replicates were performed for each sample. The relative expression levels were calculated using the $2^{-\Delta \Delta C T}$ method (Livak and Schmittgen, 2001) and the stable reference genes NITUB and NIRPS11 were used for normalization (Yuan et al., 2014).

\section{RNA Interference and Bioassay}

The RNA interference experiment was performed as described previously (Liu et al., 2010). Briefly, double-stranded RNA (dsRNA) was synthesized using T7 RiboMAX ${ }^{\mathrm{TM}}$ Express RNAi System (Promega) with the specific primers linked by the T7 promoter sequence at the $5^{\prime}$ end. The integrity of dsRNA was confirmed by $1.5 \%$ agarose gel electrophoresis and dsRNA concentration was determined by a spectrophotometer NanoDrop 2000C (Thermo Fisher Scientific). The conjunctive between prothorax and mesothorax was selected as the dsRNA injection site. Newly emerged females (within $24 \mathrm{~h}$ ) were anesthetized by carbon dioxide and injected with $23 \mathrm{~nL}$ dsRNA (about $100 \mathrm{ng}$ ) against the NlAKHR sequence or a

TABLE 1 | Primers used in this study.

\begin{tabular}{|c|c|}
\hline Primers & Primer sequence \\
\hline \multicolumn{2}{|l|}{ For RACE } \\
\hline AKHR-F1 & 5'-GACTTCCCCATCGACATGCAGTTCAAC-3' \\
\hline AKHR-F2 & 5'-ATGCTGCTGTCGGCTATAGGCAACTTC-3' \\
\hline AKHR-R1 & 5'-CAGACCACGCTATCTCCAACGGCAT-3' \\
\hline AKHR-R2 & 5'-AGGATGGAAAGGACGGTGAAGTTGC-3' \\
\hline \multicolumn{2}{|c|}{ For RT-PCR } \\
\hline $\mathrm{AKH}-\mathrm{F}$ & 5'-CACGGCGCAGGTCAACTTCT-3' \\
\hline $\mathrm{AKH}-\mathrm{R}$ & 5'-TTCGCATGAAGTGCAGTTGT-3' \\
\hline AKHR-F & 5'-CAAAGAACCCCAGCGTCCAG-3' \\
\hline AKHR-R & 5'-AGTCGAACTGAGCCGCGAAA-3' \\
\hline TUB-F & 5'-CACCGGCTCTGGGTTCACTT-3' \\
\hline TUB-R & 5'-GAGATGACCGGTGCGTAGGTG-3' \\
\hline RPS11-F & 5'-TCGCTCATCACGCAACAACA-3' \\
\hline RPS11-R & 5'-ACGAGACAGGCGTTGTGTCC-3' \\
\hline \multicolumn{2}{|c|}{ For qRT-PCR } \\
\hline qAKH-F & 5'-CССTTCTGATGGCAGTCCTाTG-3' \\
\hline qAKH-R & 5'-ATGGATGCCTTGCAGCCTTCT-3' \\
\hline qAKHR-F & 5'-GCAGTGCTGAAGCCGATGAA-3' \\
\hline qAKHR-R & 5'-GGATGAGCCTGCACCCTGAA-3' \\
\hline qTUB-F & 5'-ACTCGTTCGGAGGAGGCACC-3' \\
\hline qTUB-R & 5'-GTTCCAGGGTGGTGTGGGTGGT-3' \\
\hline qRPS11-F & 5'-CCGATCGTGTGGCGTTGAAGGG-3' \\
\hline qRPS11-R & 5'-ATGGCCGACATTCTTCCAGGTCC-3' \\
\hline \multicolumn{2}{|c|}{ For dsRNA synthesis } \\
\hline AKHR-Fi & 5'-ggatcctaatacgactcactataggg TTCACCGTCCTTCCATCCTC-3' \\
\hline AKHR-Ri & 5'-ggatcctaatacgactcactatagggGAATCCTAAACTGGACCGACG-3' \\
\hline GFP-Fi & 5'-ggatcctaatacgactcactatagggAAGGGCGAGGAGCTGTTCACCG-3' \\
\hline GFP-Ri & 5'-ggatcctaatacgactcactatagggCAGCAGGACCATGTGATCGCGC-3' \\
\hline
\end{tabular}

control dsRNA designed against a green fluorescent protein (GFP) gene (ACY56286) using a Nanoject II microinjection device (Drummond Scientific, Broomall, PA, United States). The knockdown efficiency of NlAKHR was measured at 24 and $48 \mathrm{~h}$ after dsRNA injection using RT-PCR and qRT-PCR as describe above. Females injected with dsRNA were starved and the number of dead females was counted every $8 \mathrm{~h}$. One hundred individuals were used in each set of repetition and three independent biological replicates were performed in RNAi experiments.

\section{NIAKH Treatment}

$\mathrm{NlAKH}$ (pQVNFSPNW-NH $\mathrm{N}_{2}$ ) was chemically synthesized (GenScript Biotech Inc., Nanjing, China) and dissolved in dimethyl sulfoxide (DMSO). In the present study, $20 \mathrm{pmol}$ of $\mathrm{NlAKH}$ was injected twice daily into newly emerged females (within $24 \mathrm{~h}$ ) using a Nanoject II microinjection device (Drummond Scientific). Females injected with the same volume of DMSO were used as experimental controls. The effects of $\mathrm{NlAKH}$ injection on the mobilization of lipid reserves were measured on the third day after treatment. Hemolymph and fat bodies were collected from thirty females in each replicate and three independent biological replicates were performed.

\section{Lipid Determination}

Lipids were extracted as described previously, with moderate modification (Lorenz, 2003; Konuma et al., 2012). Briefly, hemolymph or fat bodies dissected from thirty females were mixed with $100 \mu \mathrm{L}$ of $75 \%$ methanol, containing $10 \mathrm{mg}$ of sodium sulfate and homogenized in a $300 \mu \mathrm{L}$ mixture of chloroform/methanol (1:1), and then centrifuged at $12000 \times g$ at $4^{\circ} \mathrm{C}$ for $10 \mathrm{~min}$. The supernatant was removed into a new tube and mixed with $150 \mu \mathrm{L}$ of chloroform and $250 \mu \mathrm{L}$ of $1 \mathrm{M} \mathrm{NaCl}$, and the solvent was evaporated using a vacuum with centrifugation. The lipids from the organic layer were used for lipid quantification using a standard sulfo-phospho-vanillin method (Van, 1985; Xu et al., 2013).

Extracted lipids in chloroform/methanol $(2 \mu \mathrm{L})$ mixed with $1 \mathrm{~mL}$ of sulfuric acid were heated at $100^{\circ} \mathrm{C}$ for $10 \mathrm{~min}$ and then cooled to room temperature, followed by adding $1 \mathrm{~mL}$ of vanillin reagents $(0.2 \%$ vanillin in $67 \%$ ortho-phosphoric acid). Samples were measured at $540 \mathrm{~nm}$ using a NanoDrop 2000C spectrophotometer (Thermo Fisher Scientific), and lipid content was calculated against a lipid standard (cholesterol). Lipids derived from hemolymph and fat bodies were quantified as 1,2-diacylglycerol (DAG) and TAG, respectively.

\section{Nile-Red Staining}

Fat bodies were dissected in the precooled PBS buffer $(\mathrm{pH}$ 7.4) and the adherent tissues were carefully removed with forceps as thoroughly as possible under a stereomicroscope (SMZ745, Nikon). The dissected fat bodies were fixed with $4 \%$ paraformaldehyde on a glass slide for $2 \mathrm{~h}$ at room temperature and then washed with PBS for three times $(3 \mathrm{~min} \times 5 \mathrm{~min})$. For lipid staining, fat bodies were submerged in Nile red solution [ $1 \mu \mathrm{L}$ of Nile red $(1 \mathrm{mg} / \mathrm{mL})$ in $100 \mu \mathrm{L}$ of PBS] and visualized using a Ti-S inverted fluorescence microscope (Nikon) within $2 \mathrm{~h}$. 


\section{Statistical Analysis}

Results were presented as means \pm SE (standard error) based on at least three independent biological replications. Differences between two groups were analyzed by Student's $t$-test. One-way ANOVA followed by Duncan's multiple comparison was used for the comparison among more than two different conditions. $P$-values less than $0.05\left(^{*}\right)$ or $0.01\left({ }^{* *}\right)$ were considered to be statistically significant. Graphical representations and all statistical analyses were performed using GraphPad Prism 7.0 software (GraphPad Software, San Diego, CA, United States).

\section{RESULTS}

\section{Gene Identification and Phylogenetic Analysis}

NlAKH (AB817235) was identified in a previous study (Tanaka et al., 2014) and NlAKHR (MH238458) was cloned in this work. The full-length sequence of NlAKHR is $1610 \mathrm{bp}$, including a 1212 bp open reading frame (ORF) that encodes a protein consisting of 403 amino acid residues. No signal peptide cleavage was found in NlAKHR. Seven transmembrane domains (TM) were identified in NlAKHR, which indicates that this protein is a member of the GPCR superfamily (Figure 1). Alignment of NlAKHR with other insect AKHRs showed that the transmembrane domain regions possessed particularly high conservation. The conserved sequence of DRY (positions 153155), which was suggested to participate in AKHR signaling transduction and G-protein coupling, was identified (Wess, 1997). Global alignment showed that NlAKHR possessed a highly conserved structure and sequence homologies with other known insect AKHRs, and the deduced amino acid sequence of NlAKHR was approximately $60 \%$ identical to those described previously. Phylogenetic analysis showed that NlAKHR and AKHRs from other insect species clustered in a group, and NlAKHR is most closely related to G. bimaculatus homologs (GbAKHR) (Figure 2).

\section{Spatial and Developmental Expression Profiles of NIAKHR and NIAKH}

Expression patterns of NlAKHR and NlAKH in different tissues and developmental stages were determined by RT-PCR and

ATGAACGTGTCTGCTGGAGGTGACGAGCTACAAGGATCGCAAAATGGCTCGCTATCGGAGTGCGGCCCGCAGCAATGGCCGCACCTGCAG 90

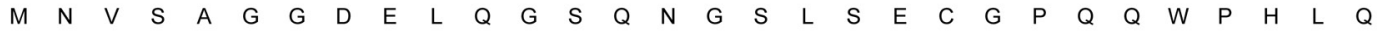
CTGTTCCCCCACTGGCACCAGTGGTGCGTCAACTCCACCTTCACCTTCGACTTCCCCATCGACATGCAGTTCAACGACGGGCATCGCCTC 180

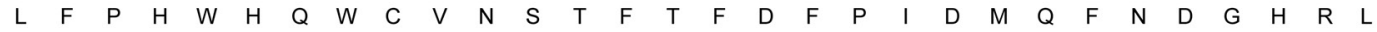
TCCATCATCGCCTACTCCACCCTCATGCTGCTGTCGGCTATAGGCAACTTCACCGTCCTTTCCATCCTCATTCGGCGCAGGCGCAACAAC 270

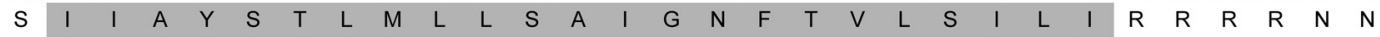
TM1 ATCAACAACATGCTTATACACCTTGCCATCGCCGATTTGCTCGTGACGTTCTTGCTGATGCCGTTGGAGATAGCGTGGTCTGCGACAGTG 360

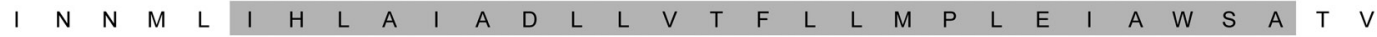
TM2

ATGTGGTGGGCTGGCGACGCTTGGTGTCGCGTCACAGCTTTCTTTCGCACCTTCGGTCTCTTCCAGTCCTCCTTCGTCCTTGTCTGCATC 450

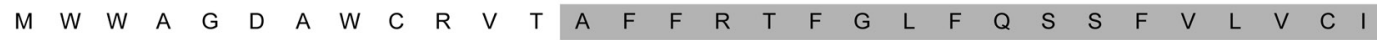
TM3

AGTATCGATAGGTATTTTGCAGTGCTGAAGCCGATGAATCATCTATCAGATGTGGACAGAAGAGGCAAGATAATGCTCAGCTGTGCTTGG 540

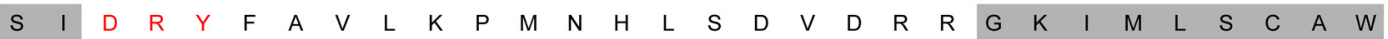
TM4 ATTGGCTCAGTCTTGTGCAGTCTACCGCAGATGCTGGTATTCAGGGTGCAGGCTCATCCGTACGTGCCATGGTTTGAGCAGTGCATCACC 630

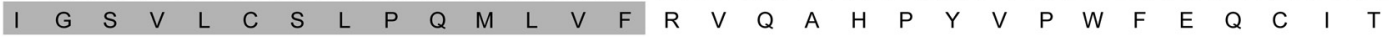
ATATGGTTTGCGCAGGACGCAAAGAACCCCAGCGTCCAGGAATTCGTGTACTTTGTGTTCGGAATGCTCATGATGTACTGTATTCCCTTC 720

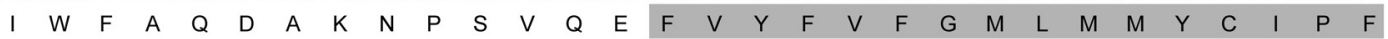
TM5

CTTGTCATACTCTTCTGCTATGCCTCGATTCTAGgCGAGATCTACCGGCACTTAAGAGAAGATAAATCGGATAGATTTCGTCGGTCCAGT 810

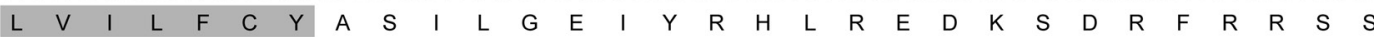
TTAGGATTCCTGGGCAAAGCTAAAGGAAGAACACTAAAAACGACTGTCATTATAGTTGTAGTGTTCCTCATCTGTTGGACACCTTATTAC 900

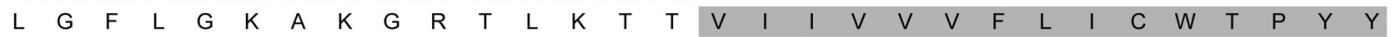
TM6 TTCATGTCTCTGTGGCACTTCTTCGACCACAAGGGCTCACAGAAGGTGGACCAGAAGCTGCAGAAAGGGCTGTACATATTCGCATGCACC 990

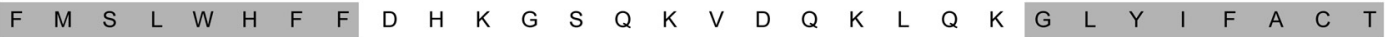
TM7

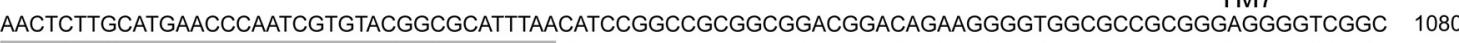

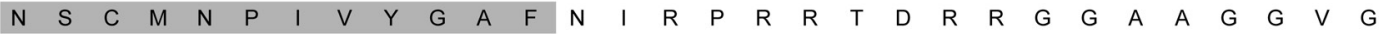
GGACAGCGGCGGCGGCCAATCCATCAGTTATCAGACGACTCAATCCAGATTTCGCGGCTCAGTTCGACTGTTTCGAATGGCTCTCAAACG 1170

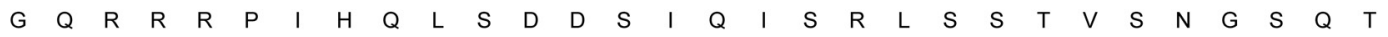
CAGCGCAATGATACTAAgCAGTCCGTTGTGTTCAGTCTGTAG

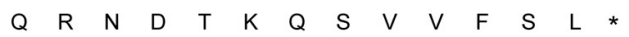

FIGURE 1 | Nucleotide and its deduced amino acid sequence of Nilaparvata lugens adipokinetic hormone receptor (NIAKHR). The numbering for each sequence is marked on the right. Seven predicted transmembrane (TM) regions 1-7 are shaded in gray. Red: amino acid residues predicted for AKHR signaling transduction and G-protein coupling. The stop codon is labeled by an asterisk. 


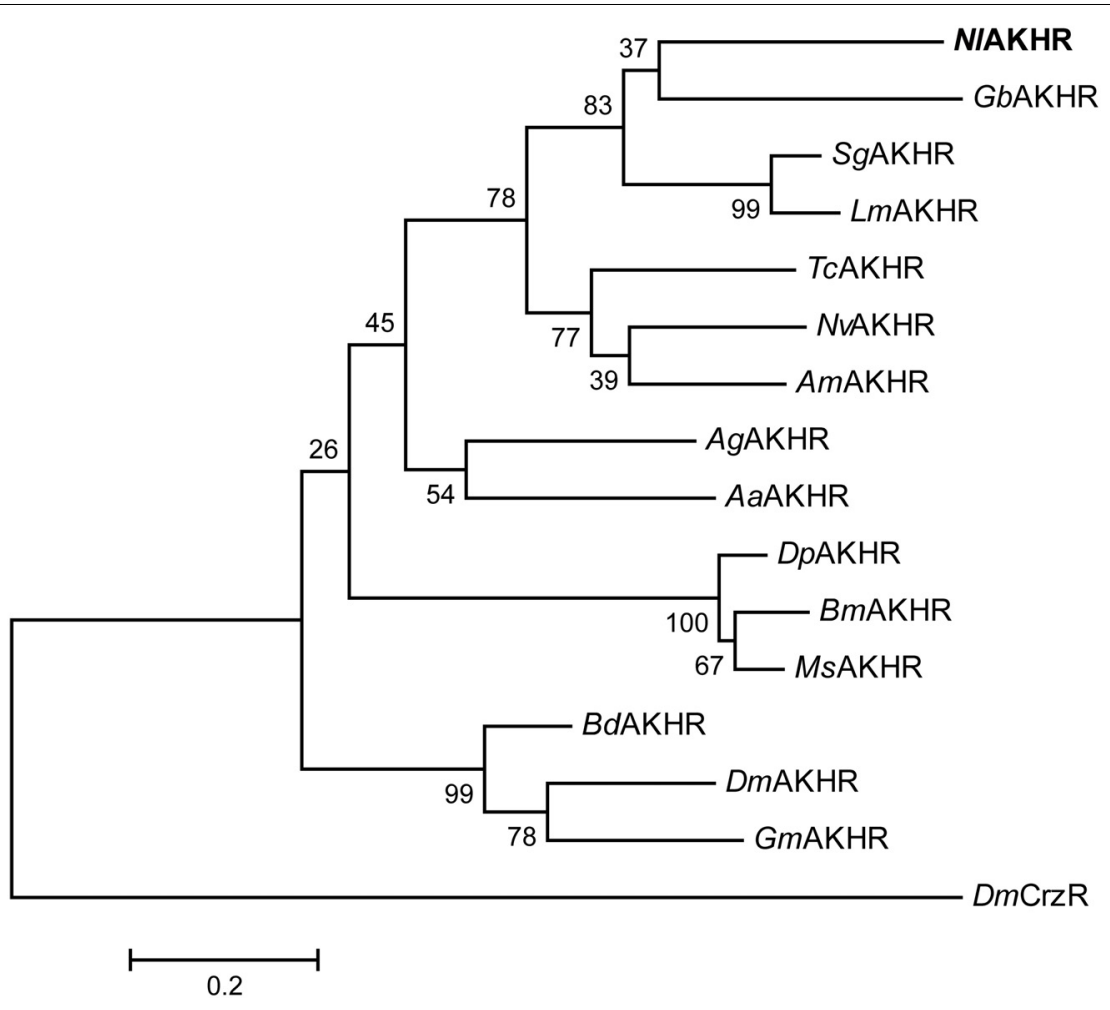

FIGURE 2 | Phylogenetic tree of NIAKHR and other insect AKHRs. The amino acid sequences of AKHR from N. lugens (NIAKHR, MH238458), Aedes aegypti (AaAKHR, CAY77164), Anopheles gambiae (AgAKHR, ABD60146), Apis mellifera (AmAKHR, NP_001035354), Bactrocera dorsalis (BdAKHR, AQX83416), Bombyx mori (BmAKHR, NP_001037049), Drosophila melanogaster (DmAKHR, NP_995639), Danaus plexippus (DpAKHR, OWR46881), Glossina morsitans (GmAKHR, AEH25943), Gryllus bimaculatus (GbAKHR, ADZ17179), Locusta migratoria (LmAKHR, ANW09575), Manduca sexta (MsAKHR, AEH25943), Nasonia vitripennis (NvAKHR, NP_001161243), Schistocerca gregaria (SgAKHR, AVG47955), and Tribolium castaneum (TCAKHR, NP_001076809) were aligned using the ClustalW program. The tree was constructed by MEGA 6 using the Maximum Likelihood (ML) method with 1000 bootstrap replicates. D. melanogaster Corazonin receptor (DmCrzR, AF373862) was used as external group. The scale bar represents 0.2 amino acid substitutions per site.

qRT-PCR. For the tissue-specific expression patterns, different tissues were dissected from 3-day-old females. The highest transcript level of $N l A K H R$ was detected in fat body, followed by head and epidermis, with lower levels in the midgut and ovary (Figure 3A). It should be noted that part of the NlAKHR expression found in the head and epidermis of female $N$. lugens is attributable to the existence of fat body cells in these two organs. Developmental expression profile results showed that NlAKHR was highly expressed in adult males and females, with relatively lower levels in nymphal stages (Figure 3B). In contrast, $N l A K H$ was exclusively expressed in the head, but not in the other tissues (Figure 4A). The highest expression of NlAKH was observed in adult males, with the lower levels in other nymphal stages and females (Figure 4B).

\section{Effects of NIAKHR Knockdown and NIAKH Injection on Female Starvation Resistance, Lipid Content and Body Weight}

Compared to normally fed females, the gene expression levels of NlAKHR increased significantly by 1.4-, 2.4-, 2.4-, and 3.1fold at $6(P=0.035), 12(P=0.028), 24(P=0.001)$ and
$48 \mathrm{~h}(P<0.001)$ after starvation, respectively (Figure 5A). The expression levels of NIAKH were elevated significantly, by 1.8 -fold at $48 \mathrm{~h}$ after starvation $(P=0.015)$ (Figure 5B). To further confirm the roles of the AKH signaling system in lipid metabolism and starvation resistance in N. lugens, the dsRNA-mediated knockdown of NlAKHR was performed. The $d s A K H R$ treatment of females resulted in a reduction of $N I A K H R$ transcripts by $68.1 \%(P=0.005)$ and $81.9 \%(P<0.001)$ compared to $d s G F P$-injected controls at 24 and $48 \mathrm{~h}$ after dsRNA injection, respectively (Figure 6A). Females under starvation conditions after NIAKHR knockdown lived $24 \mathrm{~h}$ longer than the $d s G F P$ injected controls $(P=0.0027)$ (Figure 6B). AKH injection significantly reduced the median lifespan of females by $40 \%$ after starvation compared to DMSO-injected controls $(P=0.0226)$ (Figure 6C). Knockdown of NlAKHR led to an excessive accumulation of TAG (1.6-fold) $(P=0.004)$ and glyceride (1.3fold) $(P=0.047)$ compared to the $d s G F P$-injected controls at $48 \mathrm{~h}$ after dsRNA injection. Conversely, AKH injection significantly depleted the TAG and glyceride contents of starved females by $43.2 \%(P=0.016)$ and $55.6 \%(P=0.002)$, respectively (Figures 6D,E). Females lacking AKHR function showed signs of obesity, accumulating $12.7 \%$ more body weight compared to the $d s G F P$-injected controls $(P=0.036)$ (Figure 6F). On the contrary, 
A
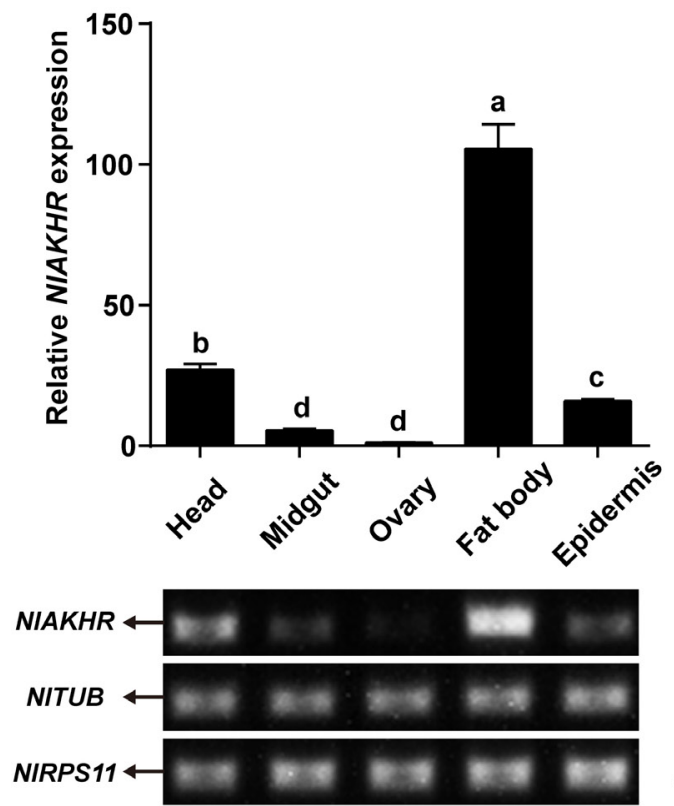

B
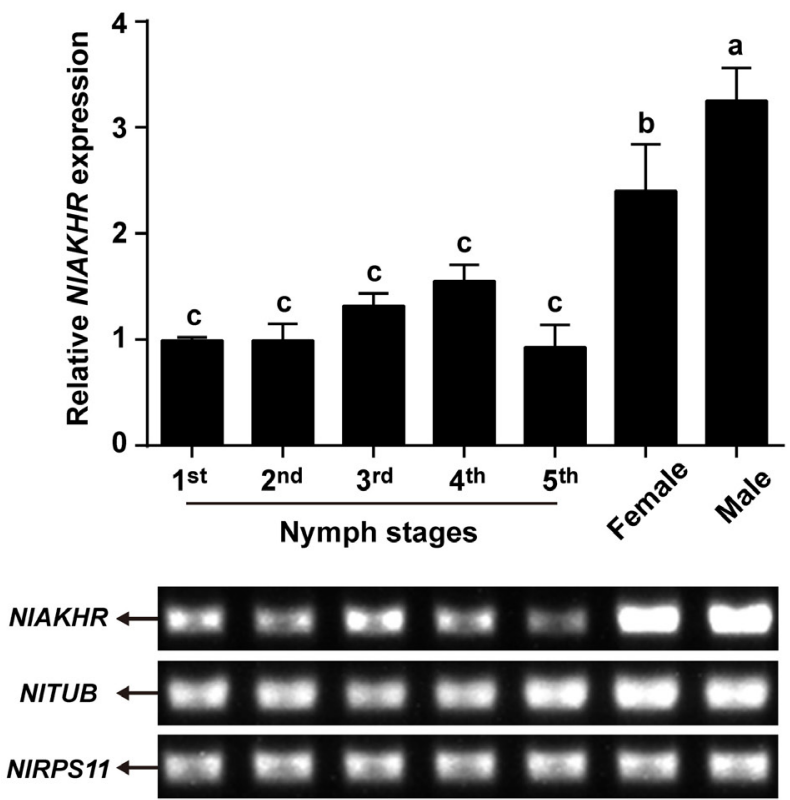

FIGURE 3 | Expression analyses of NIAKHR in different tissues and developmental stages. (A) qRT-PCR and RT-PCR analyses of NIAKHR expression levels in different tissues from 3-day-old adult females. (B) qRT-PCR and RT-PCR analyses of NIAKHR expression levels in fat bodies from the first instar nymph to adults. Results are represented as means \pm SE of three independent samples, and samples are normalized to TUB and RPS11 expression levels. Different lowercase letters represent significant difference of NIAKHR levels among various tissues and developmental stages determined by one-way ANOVA followed by Duncan's multiple comparison test $(P<0.05)$.

A

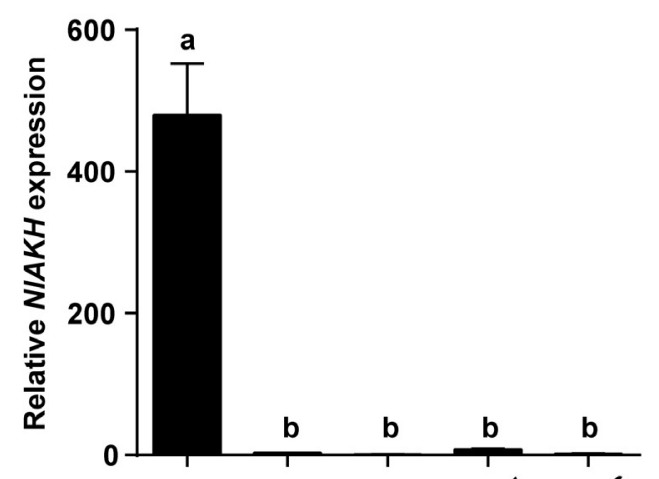

B

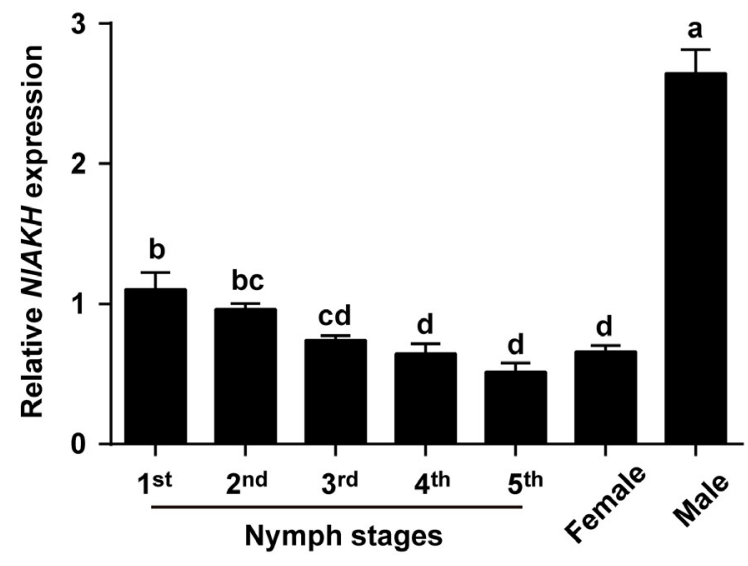

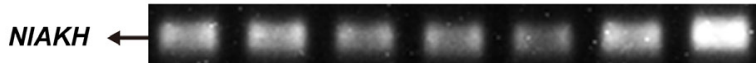

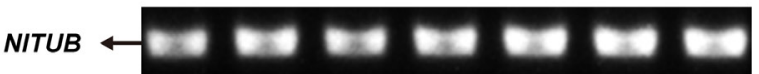

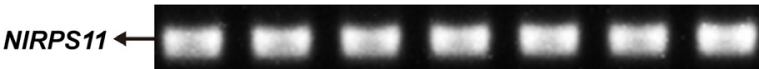

FIGURE 4 | Expression analyses of NIAKH in different tissues and developmental stages. (A) qRT-PCR and RT-PCR analyses of NIAKH expression levels in different tissues from 3-day-old adult females. (B) qRT-PCR and RT-PCR analyses of NIAKH expression levels in heads from the first instar nymph to adults. TUB and RPS11 were used as normalization controls. Results are displayed as means $\pm \mathrm{SE}$ of three independent replicates. Different lowercase letters represent significant difference of NIAKH levels among various tissues and developmental stages determined by one-way ANOVA followed by Duncan's multiple comparison test $(P<0.05)$. 
A

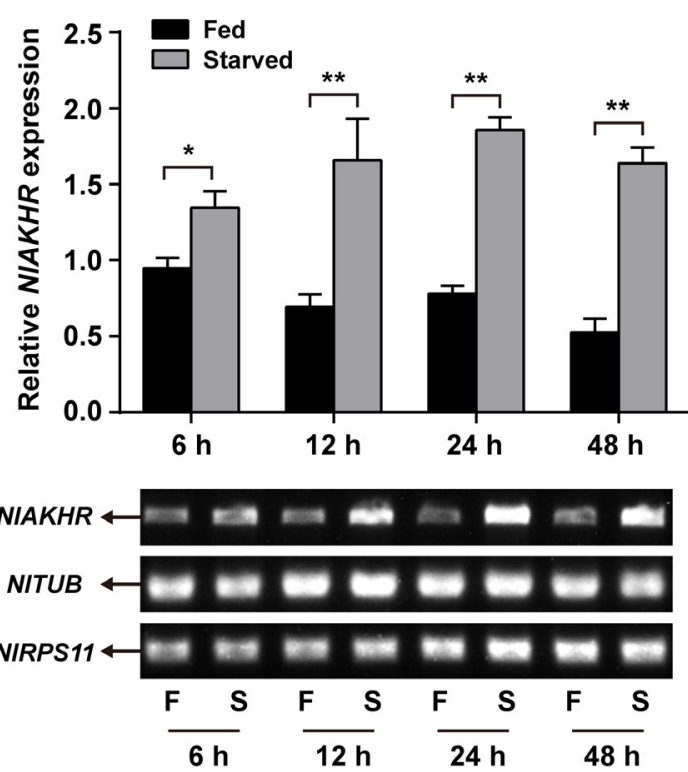

B
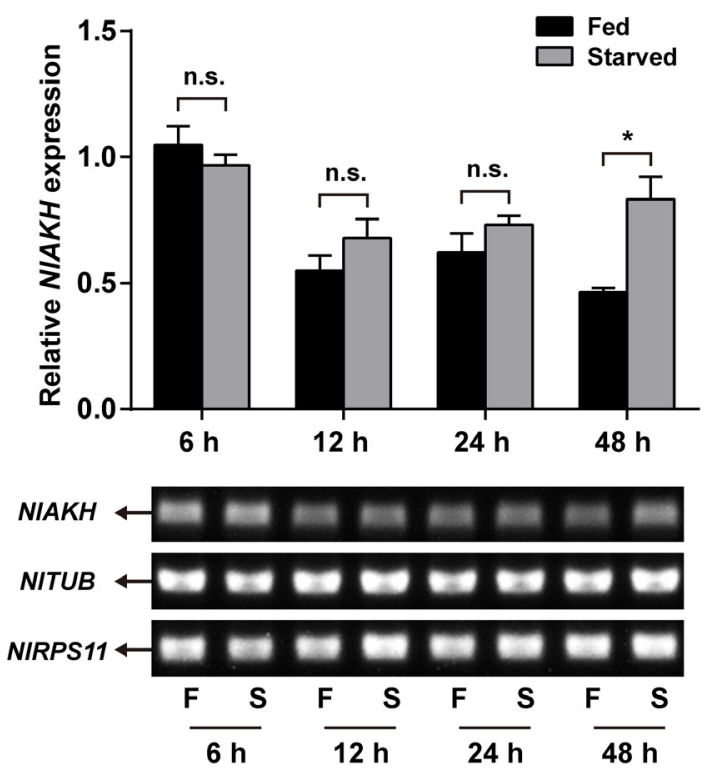

FIGURE 5 | Effects of starvation on the expression of NIAKHR (A) and NIAKH (B). Newly emerged females (within 24 h) were fed (F) or starved (S) for different hours. Differences between mRNA levels were determined by qRT-PCR and RT-PCR. TUB and RPS11 were used as internal reference controls. Results are displayed as means \pm SE of three independent replicates and asterisks indicate significant differences between the fed and starved groups at the same time point $(* P<0.05$ and $\left.{ }^{* *} P<0.01\right)$ by Student's $t$-test. n.s. represents no significant difference $(P>0.05)$.

$\mathrm{AKH}$ injection, which accelerated lipid mobilization, resulted in a $21.9 \%$ reduction of body weight and produced slim females $(P=0.001)$ (Figure 6G).

\section{Effects of NIAKHR Knockdown and NIAKH Injection on Lipid Mobilization}

Knockdown of NlAKHR resulted in reduced levels of DAG (34.6\% decrease) $(P<0.001)$ in the hemolymph compared with the $d s G F P$-injected controls. Conversely, the increased lipid levels in the hemolymph were confirmed by the results that AKH injection led to a critical accumulation of DAG (1.45fold increase) in the hemolymph $(P=0.002)$ (Figure 7A). TAG levels in the fat body of NlAKHR-silenced females significantly increased compared to that of $d s G F P$-treated controls (1.9-fold increase) $(P<0.001)$, whereas AKH injection resulted in a $39.1 \%$ reduction of TAG content in the fat body $(P<0.001)$ (Figure 7B). To measure the stored lipid reserves in the fat body, Nile-red staining was performed to visualize the lipid droplets. As shown in Figure 7C, the size and number of visualized lipid droplets critically increased after NlAKHR knockdown, whereas AKH injection resulted in a critical reduction of lipid storage droplets in the fat body compared with the DMSO-treated controls.

\section{DISCUSSION}

Two AKHRs, which only differ at their C-terminus by containing phosphorylation sites for GPCR internalization, were identified in Aedes aegypti (Kaufmann et al., 2009) and G. morsitans (Attardo et al., 2012). However, in the present study, only a single copy gene that exhibits a high degree of homology to other AKHRs, was identified from N. lugens. Several lines of evidence support the idea that the putative NlAKHR reported here is indeed an insect AKHR. Firstly, seven transmembrane domains that are involved in GPCR ligand binding and receptor activation are functionally conserved in NlAKHR. In addition, it contains specific amino acid motifs typical for the GPCR family (Gonzalez et al., 2012). Secondly, the isolated NlAKHR was highly analogous to other receptors that have been functionally characterized as AKHR from various insect species. Furthermore, NlAKHR knockdown resulted in decreased levels of circulating DAG in the hemolymph and an accumulation of TAG in the fat body. Our results clearly demonstrate that AKHR is critical for the maintenance of energy homeostasis, possibly due to the structural and functional conservation of the AKH signaling system in the regulation of lipolysis.

Adipokinetic hormone neuropeptides are primarily synthesized in the corpora cardiaca and are responsible for lipid mobilization during energy-demanding processes in a wide diversity of insect species (Auerswald et al., 2005; Auerswald and Gäde, 2006). In D. melanogaster, only a single AKH gene was identified (Gäde, 2010), while two AKH precursors that possess a similar structure typical of the $\mathrm{AKH}$ family were identified from the tsetse fly G. morsitans (Attardo et al., 2012) and the mosquito A. aegypti (Kaufmann et al., 2009). Both tsetse AKH peptides are the cognate ligands of $G m A K H R$, indicating these two $A K H$ genes derived from recent gene duplication (Caers et al., 2016). NlAKH is exclusively expressed in the head of $N$. lugens, as has been demonstrated to be present in several insect species (Siegert, 1999; Kaufmann and Brown, 2006; 


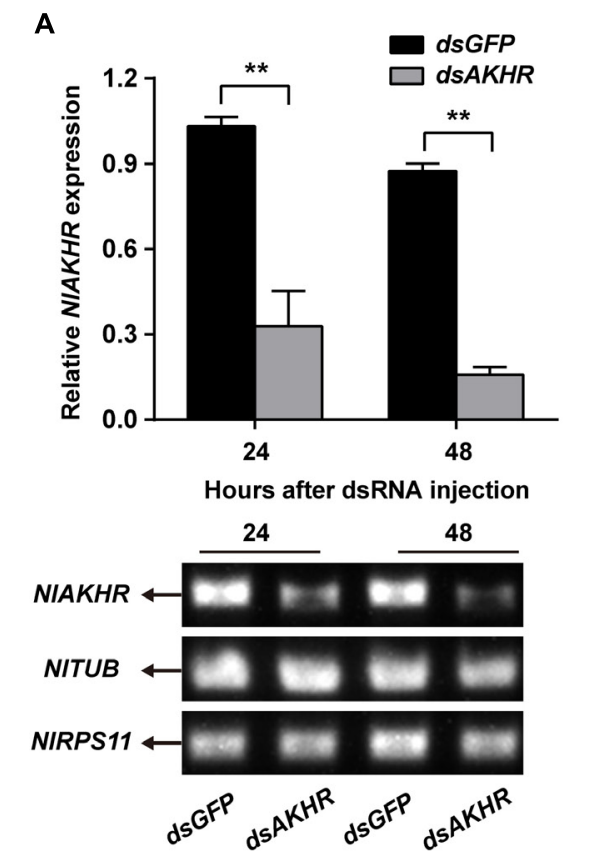

F

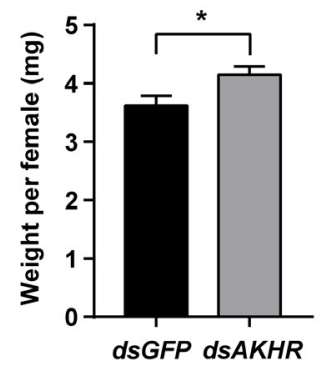

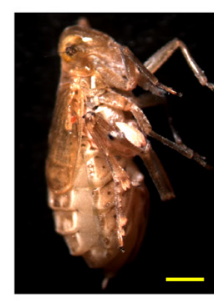

dsGFP
B

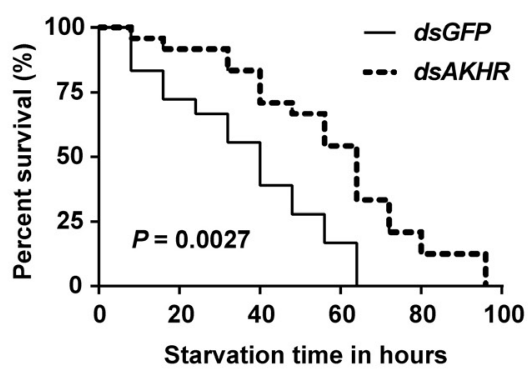

D

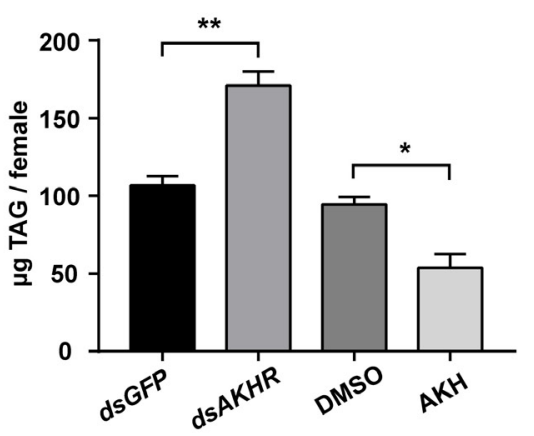

G

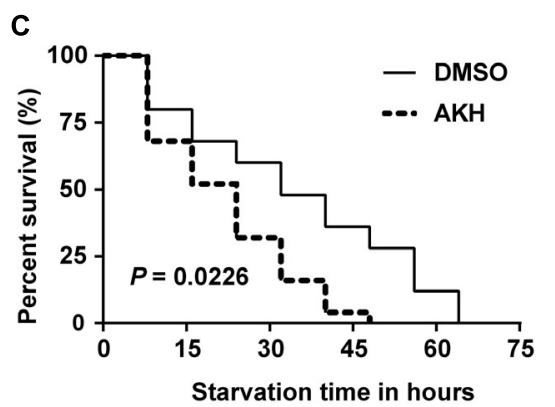

E

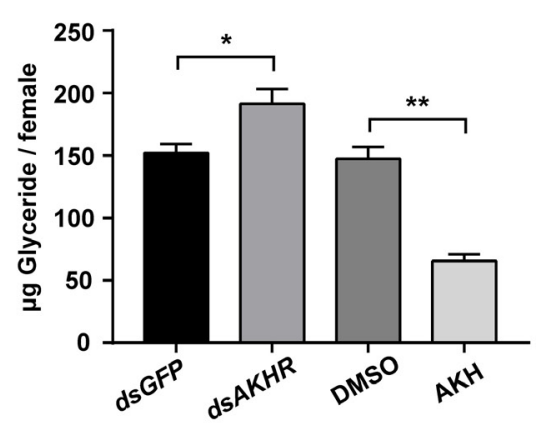

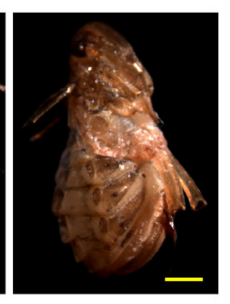

dsAKHR

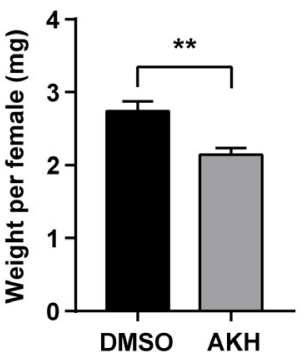

DMSO AKH

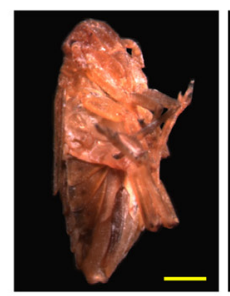

DMSO

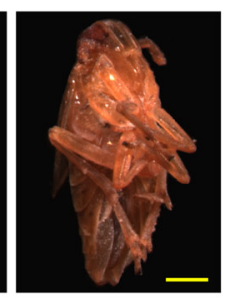

AKH

FIGURE 6 | Effects of NIAKHR knockdown and AKH injection on the starvation resistance, whole body lipids and body weight. For RNAi, newly emerged females (within $24 \mathrm{~h}$ ) were injected with $100 \mathrm{ng}$ of dsRNA against NIAKHR (dsAKHR) or with a control dsRNA (dsGFP). For AKH treatment, newly emerged females were injected with $20 \mathrm{pmol}$ of AKH or DMSO (control). (A) Fat bodies were dissected at 24 and $48 \mathrm{~h}$ after dsRNA injection. Differences between NIAKHR expression levels were determined by qRT-PCR and RT-PCR. TUB and RPS11 were used as internal reference controls. Survival rates for dsAKHR-injected females (B) and $\mathrm{AKH}$-treated females (C) under starvation condition were analyzed with Kaplan-Meier plot with a log rank test. TAG (D) and glyceride (E) contents in the whole body of $N$. lugens were determined at $48 \mathrm{~h}$ after injection. (F) Body weight of females was measured on the sixth day after dsRNA injection ( $n=23-28)$. (G) Body weight of females was measured on the third day after AKH treatment $(n=12)$. Results are represented as means \pm SE of three independent replicates and asterisks indicate significant differences $\left({ }^{*} P<0.05\right.$ and $\left.{ }^{*} P<0.01\right)$ by Student's $t$-test. Scale bar, $0.5 \mathrm{~mm}$.

Kaufmann et al., 2009). We also observed the highest expression levels of NlAKH in adult males of $N$. lugens, which indicates an interesting role for $\mathrm{AKH}$ in the energy mobilization associated with flight and, in particular, the reproduction of males. These high expression levels suggest a conserved function of the $\mathrm{AKH}$ signaling system in the regulation of lipid mobilization and energy homeostasis. NIAKHR was mostly expressed in the fat body of adult females, as also observed in A. aegypti (Kaufmann et al., 2009), Anopheles gambiae (Kaufmann and Brown, 2006), D. melanogaster (Staubli et al., 2002), and Manduca sexta (Ziegler et al., 2011). This corresponds with the main function of $\mathrm{AKH}$ on lipid and carbohydrate mobilization from the fat body under conditions of high energy demand (Marco et al., 2013). In insects, the fat body is the main organ for lipid storage and energy utilization (Arrese and Soulages, 2010). The highest expression level in the fat body also suggests a conserved role of AKHR in the regulation of energy mobilization in $N$. lugens.

Many studies have demonstrated that the AKH signaling system plays an important role in lipid mobilization. Nutritional status, either feeding or starvation, can significantly affect the expression of AKH signaling system components. For example, $A K H R$ transcript levels decreased after protein meals and it is likely that TAG synthesis may be stimulated and lipolysis may be inhibited in the blood-fed mosquito A. gambiae (Kaufmann and Brown, 2006) and liver-fed flesh fly S. crassipalpis (Bil et al., 2016). It also corresponds well with the decrease of AKH neuropeptides observed in the liver fed S. crassipalpis (Bil et al., 2014). Here, we found that starvation induces the expression of $A K H$ and its receptor $A K H R$, indicating that the $\mathrm{AKH}$ signaling system is involved in the regulation of starvation resistance. 
A

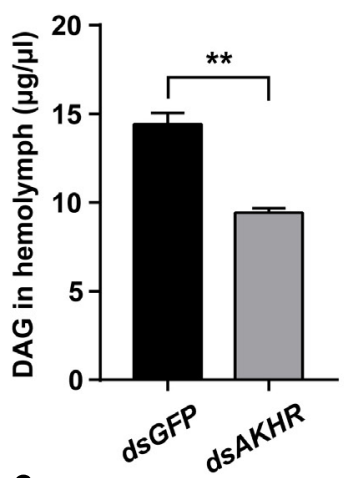

C

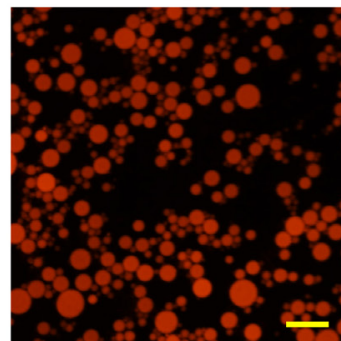

dsGFP
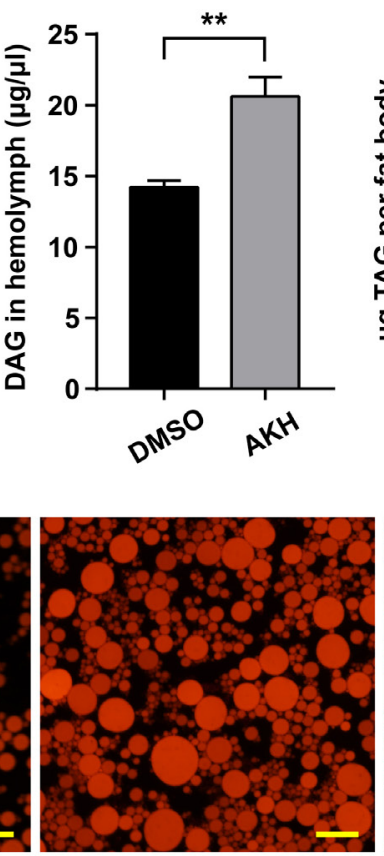

dsAKHR
B
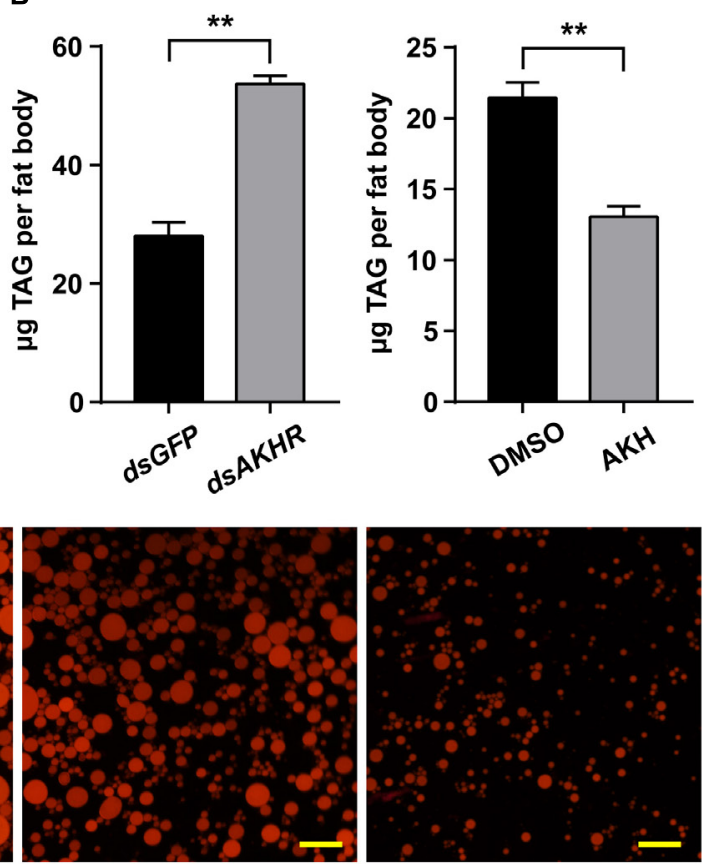

DMSO

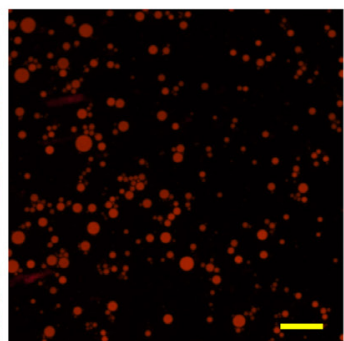

AKH

FIGURE 7 | Effects of NIAKHR knockdown and AKH injection on the TAG mobilization. For RNAi, newly emerged females (within 24 h) were injected with 100 ng of dsRNA against NIAKHR (dsAKHR) or with a control dsRNA (dsGFP). For AKH treatment, 20 pmol of NIAKH was injected twice daily into newly emerged females, and females injected with the same volume of DMSO were used as experimental controls. The amounts of DAG in the hemolymph (A) and TAG in the fat body (B) were determined on the third day after treatment. Results are represented as means \pm SE of three independent replicates and asterisks indicate significant differences $\left(* P<0.05\right.$ and $\left.{ }^{*} P<0.01\right)$ by Student's $t$-test. DAG, diacylglycerol; TAG, triacylglycerol. (C) Representative images from fat bodies after Nile red staining. Scale bar, $20 \mu \mathrm{m}$.

It seems likely that the higher abundance of $\mathrm{AKH}$ and $\mathrm{AKHR}$ stimulates the use of stored lipid reserves and meets energy demands when food is unavailable. In D. melanogaster, AKHR mutant flies possessed high levels of lipids at the time of death, and this is likely due to the inability of flies to mobilize stored lipids under starvation conditions (Grönke et al., 2007). Our results showed that knockdown of NIAKHR resulted in extended survival during starvation and accumulated lipid reserves, as previously observed in D. melanogaster (Grönke et al., 2007) and G. bimaculatus (Konuma et al., 2012). Conversely, the rate of lipolysis under starvation conditions accelerated and starvation resistance decreased after $\mathrm{AKH}$ exposure. Since the lipid reserves deposited in the fat body are the main energy resources under food-deprived conditions, it is likely that AKH signaling systemmediated lipolysis is essential for starvation resistance. NlAKHR knockdown females appear incapable of mobilizing lipid reserves, resulting in an obese phenotype. Similar results have been obtained in the cricket G. bimaculatus, wherein knockdown of $A K H R$ resulted in an increase of TAG in the fat body and a decrease of DAG in the hemolymph (Konuma et al., 2012). AKHR mutants of $D$. melanogaster also accumulated high levels of TAG in the fat body (Grönke et al., 2007; Bharucha et al., 2008). These results support the idea that TAG mobilization is closely related to AKHR expression, and the AKH signaling system is critical for maintaining energy homeostasis. Here, we demonstrate that knockdown of NIAKHR increased the TAG levels in the fat body and decreased circulating DAG contents in the hemolymph. Conversely, NlAKH-treated females are slim with a severe reduction in stored lipid levels. This phenotype is a probable result of reduced fat body lipid reserves during starvation as $\mathrm{AKH}$ accelerates lipid mobilization. The inability to accumulate lipid reserves after $\mathrm{AKH}$ exposure was demonstrated in the locust Schistocerca gregaria, and the mosquito A. aegypti (Ziegler, 1997; Gokuldas et al., 2010). In addition, the exposure of $\mathrm{NlAKH}$ also affected the distribution of lipids, decreasing TAG levels in the fat body while increasing circulating DAG levels in the hemolymph. Based on these results, we speculate here that lipid mobilization in the fat body is tightly regulated by the AKH signaling system, which is essential in adjusting body lipid homeostasis and ensuring energy supplementation during starvation in N. lugens.

Two lipolytic systems, including the AKH/AKHR signaling system and brummer lipase system, are conserved in N. lugens, as has been shown in D. melanogaster (Grönke et al., 2005, 2007) and G. morsitans (Attardo et al., 2012). These two pathways are somewhat functionally redundant in regard to lipid mobilization; that is, when one pathway is suppressed the other may account for partial compensation. Our previous studies have demonstrated that brummer lipase plays an important role in maintaining hemolymph lipid levels during the starvation 
period (Zhou et al., 2018a). Given the scale of lipid mobilization required for survival during periods of nutritional stress, it appears that both the AKH/AKHR and brummer pathways are critical for lipid mobilization and starvation resistance (Grönke et al., 2005, 2007). The combination of these two lipolytic systems in $N$. lugens provides the flexibility to resist various kinds of stress by utilizing the strengths of one system to compensate for the weakness of the other. The molecular mechanisms that govern the cross-talk between these two lipolytic pathways in the regulation of lipid homeostasis and starvation resistance are the focus of research in our laboratory.

\section{AUTHOR CONTRIBUTIONS}

$\mathrm{KL}$ and $\mathrm{QZ}$ designed the research and wrote the paper. $\mathrm{XZ}, \mathrm{XC}$, $\mathrm{YL}, \mathrm{WL}$, and YC performed the experiments and analyzed the

\section{REFERENCES}

Alves-Bezerra, M., Paula, I. F. D., Medina, J. M., Silva-Oliveira, G., Medeiros, J. S., Gäde, G., et al. (2016). Adipokinetic hormone receptor gene identification and its role in triacylglycerol metabolism in the blood-sucking insect Rhodnius prolixus. Insect Biochem. Mol. Biol. 69, 51-60. doi: 10.1016/j.ibmb.2015. 06.013

Arrese, E. L., Flowers, M. T., Gazard, J. L., and Wells, M. A. (1999). Calcium and cAMP are second messengers in the adipokinetic hormone-induced lipolysis of triacylglycerols in Manduca sexta fat body. J. Lipid Res. 40, 556-564.

Arrese, E. L., and Soulages, J. L. (2010). Insect fat body: energy, metabolism, and regulation. Annu. Rev. Entomol. 55, 207-225. doi: 10.1146/annurev-ento112408-085356

Attardo, G. M., Benoit, J. B., Michalkova, V., Yang, G., Roller, L., Bohova, J., et al. (2012). Analysis of lipolysis underlying lactation in the tsetse fly, Glossina morsitans. Insect Biochem. Mol. Biol. 42, 360-370. doi: 10.1016/j.ibmb.2012. 01.007

Auerswald, L., and Gäde, G. (2006). Endocrine control of TAG lipase in the fat body of the migratory locust, Locusta migratoria. Insect Biochem. Mol. Biol. 36, 759-768. doi: 10.1016/j.ibmb.2006.07.004

Auerswald, L., Siegert, K. J., and Gäde, G. (2005). Activation of triacylglycerol lipase in the fat body of a beetle by adipokinetic hormone. Insect Biochem. Mol. Biol. 35, 461-470. doi: 10.1016/j.ibmb.2005.01.010

Azeez, O. I., Meintjes, R., and Chamunorwa, J. P. (2014). Fat body, fat pad and adipose tissues in invertebrates and vertebrates: the nexus. Lipids Health Dis. 13, 1-13. doi: 10.1186/1476-511X-13-71

Bednářová, A., Kodrík, D., and Krishnan, N. (2013). Adipokinetic hormone exerts its anti-oxidative effects using a conserved signal-transduction mechanism involving both PKC and cAMP by mobilizing extra- and intracellular Ca2+ stores. Comp. Biochem. Physiol. C Toxicol. Pharmacol. 158, 142-149. doi: 10. 1016/j.cbpc.2013.07.002

Bharucha, K. N., Tarr, P., and Zipursky, S. L. (2008). A glucagon-like endocrine pathway in Drosophila modulates bothlipid and carbohydrate homeostasis. J. Exp. Biol. 211, 3103-3110. doi: 10.1242/jeb.016451

Bil, M., Broeckx, V., Landuyt, B., and Huybrechts, R. (2014). Differential peptidomics highlights adipokinetic hormone as key player in regulating digestion in anautogenous flesh fly. Sarcophaga Crassipalpis. Gen. Comp. Endocrinol. 208, 49-56. doi: 10.1016/j.ygcen.2014.08.016

Bil, M., Timmermans, I., Verlinden, H., and Huybrechts, R. (2016). Characterization of the adipokinetic hormone receptor of the anautogenous flesh fly, Sarcophaga crassipalpis. J. Insect Physiol. 89, 52-59. doi: 10.1016/j.jinsphys.2016.04.001

Brown, D. A. (2001). Lipid droplets: proteins floating on a pool of fat. Curr. Biol. 11, R446-R449. doi: 10.1016/S0960-9822(01)00257-3 data. JZ and KY revised the manuscript. All authors listed have approved the manuscript for publication.

\section{FUNDING}

This work was supported by the National Natural Science Foundation of China (31772159 and 31601634), Natural Science Foundation of Fujian Province (2017J01428), and Distinguished Youth Talent Program of Fujian Agriculture and Forestry University (xjq201722).

\section{SUPPLEMENTARY MATERIAL}

The Supplementary Material for this article can be found online at: https://www.frontiersin.org/articles/10.3389/fphys. 2018.01730/full\#supplementary-material

Caers, J., Janssen, T., Van, R. L., Broeckx, V., Van, D. A. J., Gäde, G., et al. (2016). Characterization and pharmacological analysis of two adipokinetic hormone receptor variants of the tsetse fly, Glossina morsitans morsitans. Insect Biochem. Mol. Biol. 70, 73-84. doi: 10.1016/j.ibmb.2015.11.010

Caers, J., Verlinden, H., Zels, S., Vandersmissen, H. P., Vuerinckx, K., and Schoofs, L. (2012). More than two decades of research on insect neuropeptide GPCRs: an overview. Front. Endocrinol. 3:151. doi: 10.3389/fendo.2012. 00151

Gäde, G. (2010). Peptides of the adipokinetic hormone/red pigment-concentrating hormone family: a new take on biodiversity. Ann. N. Y. Acad. Sci. 1163, 125-136. doi: 10.1111/j.1749-6632.2008.03625.x

Gäde, G., and Auerswald, L. (2003). Mode of action of neuropeptides from the adipokinetic hormone family. Gen. Comp. Endocrinol. 132, 10-20. doi: 10.1016/ S0016-6480(03)00159-X

Gäde, G., and Marco, H. G. (2013). “AKH-RPCH peptides," in Handbook of Biologically Active Peptides, ed. A. J. Kastin (San Diego, CA: Elsevier Inc.), 185-190. doi: 10.1016/B978-0-12-385095-9.00028-2

Gáliková, M., Diesner, M., Klepsatel, P., Hehlert, P., Xu, Y., Bickmeyer, I., et al. (2015). Energy homeostasis control in Drosophila adipokinetic hormone mutants. Genetics 201, 665-683. doi: 10.1534/genetics.115.178897

Gokuldas, M., Hunt, P. A., and Candy, D. J. (2010). The inhibition of lipid synthesis in vitro in the locust, Schistocerca gregaria, by factors from the corpora cardiaca. Physiol. Entomol. 13, 43-48. doi: 10.1111/j.1365-3032.1988.tb00907.x

Gonzalez, A., Cordomí, A., Caltabiano, G., and Pardo, L. (2012). Impact of helix irregularities on sequence alignment and homology modeling of G proteincoupled receptors. Chembiochem 13, 1393-1399. doi: 10.1002/cbic.2012 00189

Grönke, S., Mildner, A., Fellert, S., Tennagels, N., Petry, S., Müller, G., et al. (2005). Brummer lipase is an evolutionary conserved fat storage regulator in Drosophila. Cell Metab. 1, 323-330. doi: 10.1016/j.cmet.2005.04.003

Grönke, S., Müller, G., Hirsch, J., Fellert, S., Andreou, A., Haase, T., et al. (2007). Dual lipolytic control of body fat storage and mobilization in Drosophila. PLoS Biol. 5:e137. doi: 10.1371/journal.pbio.0050137

Hou, Q. L., Chen, E. H., Jiang, H. B., Wei, D. D., Gui, S. H., Wang, J. J., et al. (2017). Adipokinetic hormone receptor gene identification and its role in triacylglycerol mobilization and sexual behavior in the oriental fruit fly (Bactrocera dorsalis). Insect Biochem. Mol. Biol. 90, 1-13. doi: 10.1016/j.ibmb. 2017.09.006

Isabel, G., Martin, J. R., Chidami, S., Veenstra, J. A., and Rosay, P. (2005). AKH-producing neuroendocrine cell ablation decreases trehalose and induces behavioral changes in Drosophila. Am. J. Physiol. Regul. Integr. Comp. Physiol. 288, R531-R538. doi: 10.1152/ajpregu.00158.2004

Kaufmann, C., and Brown, M. R. (2006). Adipokinetic hormones in the African malaria mosquito, Anopheles gambiae: identification and expression of genes 
for two peptides and a putative receptor. Insect Biochem. Mol. Biol. 36, 466-481. doi: 10.1016/j.ibmb.2006.03.009

Kaufmann, C., Merzendorfer, H., and Gäde, G. (2009). The adipokinetic hormone system in Culicinae (Diptera: Culicidae): molecular identification and characterization of two adipokinetic hormone (AKH) precursors from Aedes aegypti and Culex pipiens and two putative AKH receptor variants from A. aegypti. Insect Biochem. Mol. Biol. 39, 770-781. doi: 10.1016/j.ibmb.2009. 09.002

Konuma, T., Morooka, N., Nagasawa, H., and Nagata, S. (2012). Knockdown of the adipokinetic hormone receptor increases feeding frequency in the two-spotted cricket Gryllus bimaculatus. Endocrinology 153, 3111-3122. doi: 10.1210/en. 2011-1533

Krogh, A., Larsson, B., Von, H. G., and Sonnhammer, E. L. (2001). Predicting transmembrane protein topology with a hidden Markov model: application to complete genomes. J. Mol. Biol. 305, 567-580. doi: 10.1006/jmbi.2000. 4315

Lee, G., and Park, J. H. (2004). Hemolymph sugar homeostasis and starvationinduced hyperactivity affected by genetic manipulations of the adipokinetic hormone-encoding gene in Drosophila melanogaster. Genetics 167, 311-323. doi: 10.1534/genetics.167.1.311

Lindemans, M., Liu, F., Janssen, T., Husson, S. J., Mertens, I., Gäde, G., et al. (2009). Adipokinetic hormone signaling through the gonadotropin-releasing hormone receptor modulates egg-laying in Caenorhabditis elegans. Proc. Natl. Acad. Sci. U.S.A. 106, 1642-1647. doi: 10.1073/pnas.0809881106

Liu, S., Ding, Z., Zhang, C., Yang, B., and Liu, Z. (2010). Gene knockdown by intro-thoracic injection of double-stranded RNA in the brown planthopper, Nilaparvata lugens. Insect Biochem. Mol. Biol. 40, 666-671. doi: 10.1016/j.ibmb. 2010.06.007

Livak, K. J., and Schmittgen, T. D. (2001). Analysis of relative gene expression data using real-time quantitative PCR and the 2- $\Delta \Delta$ CT method. Methods 25, 402-408. doi: 10.1006/meth.2001.1262

Lorenz, M. W. (2003). Adipokinetic hormone inhibits the formation of energy stores and egg production in the cricket Gryllus bimaculatus. Comp. Biochem. Physiol. B Biochem. Mol. Biol. 136, 197-206. doi: 10.1016/S1096-4959(03) 00227-6

Lu, K., Chen, X., Li, Y., Li, W., and Zhou, Q. (2018). Lipophorin receptor regulates Nilaparvata lugens fecundity by promoting lipid accumulation and vitellogenin biosynthesis. Comp. Biochem. Physiol. A Mol. Integr. Physiol. 21, 28-37. doi: 10.1016/j.cbpa.2018.02.008

Lu, K., Shu, Y., Zhou, J., Zhang, X., Zhang, X., Chen, M., et al. (2015). Molecular characterization and RNA interference analysis of vitellogenin receptor from Nilaparvata lugens (Stal). J. Insect Physiol. 73, 20-29. doi: 10.1016/j.jinsphys. 2015.01.007

Marco, H. G., Šimek, P., Clark, K. D., and Gäde, G. (2013). Novel adipokinetic hormones in the kissing bugs Rhodnius prolixus, Triatoma infestans, Dipetalogaster maxima and Panstrongylus megistus. Peptides 41, 21-30. doi: 10.1016/j.peptides.2012.09.032

Martin, S., and Parton, R. G. (2006). Lipid droplets: a unified view of a dynamic organelle. Nat. Rev. Mol. Cell Biol. 7, 373-378. doi: 10.1038/nrm1912

Mayer, R. J., and Candy, D. J. (1969). Control of haemolymph lipid concentration during locust flight: an adipokinetic hormone from the corpora cardiaca. J. Insect Physiol. 15, 611-620. doi: 10.1016/0022-1910(69)90259-5

Mccarthy, M., Duff, P., Muller, H. L., and Randell, C. (2004). Prediction of proprotein convertase cleavage sites. Protein Eng. Des. Sel. 17, 107-112. doi: 10.1093/protein/gzh013

Park, Y., Kim, Y. J., and Adams, M. E. (2002). Identification of G protein-coupled receptors for Drosophila PRXamide peptides, CCAP, corazonin, and AKH supports a theory of ligand-receptor coevolution. Proc. Natl. Acad. Sci. U.S.A. 99, 11423-11428. doi: 10.1073/pnas.162276199

Siegert, K. J. (1999). Locust corpora cardiaca contain an inactive adipokinetic hormone. FEBS Lett. 447, 237-240. doi: 10.1016/S0014-5793(99)00299-9

Staubli, F., Jorgensen, T. J., Cazzamali, G., Williamson, M., Lenz, C., Sondergaard, L., et al. (2002). Molecular identification of the insect adipokinetic hormone receptors. Proc. Natl. Acad. Sci. U.S.A. 99, 3446-3451. doi: 10.1073/ pnas.052556499
Stone, J. V., Mordue, W., Batley, K. E., and Morris, H. R. (1976). Structure of locust adipokinetic hormone, a neurohormone that regulates lipid utilisation during flight. Nature 263, 207-211. doi: 10.1038/263207a0

Tamura, K., Stecher, G., Peterson, D., Filipski, A., and Kumar, S. (2013). MEGA6: molecular evolutionary genetics analysis version 6.0. Mol. Biol. Evol. 30, 27252729. doi: $10.1093 / \mathrm{molbev} / \mathrm{mst} 197$

Tanaka, Y., Suetsugu, Y., Yamamoto, K., Noda, H., and Shinoda, T. (2014). Transcriptome analysis of neuropeptides and G-protein coupled receptors (GPCRs) for neuropeptides in the brown planthopper Nilaparvata lugens. Peptides 53, 125-133. doi: 10.1016/j.peptides.2013.07.027

Untergasser, A., Cutcutache, I., Koressaar, T., Ye, J., Faircloth, B. C., Remm, M., et al. (2012). Primer3-new capabilities and interfaces. Nucleic Acids Res. 40:e115. doi: 10.1093/nar/gks596

Van, H. E. (1985). Rapid determination of total lipids in mosquitoes. J. Am. Mosq. Control Assoc. 1, 302-304.

Van der Horst, D. J., Marrewijk, W. J. A. V., and Diederen, J. H. B. (2001). Adipokinetic hormones of insect: release, signal transduction, and responses. Int. Rev. Cytol. 211, 179-240. doi: 10.1016/S0074-7696(01)11019-3

Wess, J. (1997). G-protein-coupled receptors: molecular mechanisms involved in receptor activation and selectivity of G-protein recognition. FASEB J. 11, 346-354. doi: 10.1096/fasebj.11.5.9141501

$\mathrm{Xu}$, J., Sheng, Z., and Palli, S. R. (2013). Juvenile hormone and insulin regulate trehalose homeostasis in the red flour beetle, Tribolium castaneum. PLoS Genet. 9:e1003535. doi: 10.1371/journal.pgen.1003535

Xu, W., Huang, F. S., Hao, H. X., Duan, J. H., and Qiu, Z. W. (2006). Two serine proteases from Anopheles dirus haemocytes exhibit changes in transcript abundance after infection of an incompatible rodent malaria parasite, Plasmodium yoelii. Vet. Parasitol. 139, 93-101. doi: 10.1016/j.vetpar.2006. 02.017

Xue, J., Zhou, X., Zhang, C. X., Yu, L. L., Fan, H. W., Wang, Z., et al. (2014). Genomes of the rice pest brown planthopper and its endosymbionts reveal complex complementary contributions for host adaptation. Genome Biol. 15:521. doi: 10.1186/s13059-014-0521-0

Yuan, M., Lu, Y., Zhu, X., Wan, H., Muhammad, S., Zhan, S., et al. (2014). Selection and evaluation of potential reference genes for gene expression analysis in the brown planthopper, Nilaparvata lugens (Hemiptera: Delphacidae) using reverse-transcription quantitative PCR. PLoS One 9:e86503. doi: 10.1371/ journal.pone. 0086503

Zhou, J., Chen, X., Yan, J., You, K., Yuan, Z., Zhou, Q., et al. (2018a). Brummerdependent lipid mobilization regulates starvation resistance in Nilaparvata lugens. Arch. Insect Biochem. Physiol. 99:e21481. doi: 10.1002/arch.21481

Zhou, J., Yan, J., You, K., Chen, X., Yuan, Z., Zhou, Q., et al. (2018b). Characterization of a Nilaparvata lugens (Stål) brummer gene and analysis of its role in lipid metabolism. Arch. Insect Biochem. Physiol. 97:e21442. doi: 10.1002/arch.21442

Ziegler, R. (1997). Lipid synthesis by ovaries and fat body of Aedes aegypti (Diptera: Culicidae). Eur. J. Entomol. 94, 385-391.

Ziegler, R., Isoe, J., Moore, W., Riehle, M. A., and Wells, M. A. (2011). The putative $\mathrm{AKH}$ receptor of the tobacco hornworm, Manduca sexta, and its expression. J. Insect Sci. 11:40. doi: 10.1673/031.011.0140

Zimmermann, R., and Zechner, R. (2004). Fat mobilization in adipose tissue is promoted by adipose triglyceride lipase. Science 306, 1383-1386. doi: 10.1126/ science. 1100747

Conflict of Interest Statement: The authors declare that the research was conducted in the absence of any commercial or financial relationships that could be construed as a potential conflict of interest.

Copyright () 2018 Lu, Zhang, Chen, Li, Li, Cheng, Zhou, You and Zhou. This is an open-access article distributed under the terms of the Creative Commons Attribution License (CC BY). The use, distribution or reproduction in other forums is permitted, provided the original author(s) and the copyright owner(s) are credited and that the original publication in this journal is cited, in accordance with accepted academic practice. No use, distribution or reproduction is permitted which does not comply with these terms. 